\title{
Term Structure Models with Parallel and Proportional Shifts
}

\author{
Armerin, Frederik; Björk, Tomas; Astrup Jensen, Bjarne
}

Document Version

Final published version

Publication date:

2005

\section{License \\ CC BY-NC-ND}

Citation for published version (APA):

Armerin, F., Björk, T., \& Astrup Jensen, B. (2005). Term Structure Models with Parallel and Proportional Shifts. Institut for Finansiering, Copenhagen Business School. Working Papers / Department of Finance. Copenhagen Business School No. 2005-5

Link to publication in CBS Research Portal

\section{General rights}

Copyright and moral rights for the publications made accessible in the public portal are retained by the authors and/or other copyright owners and it is a condition of accessing publications that users recognise and abide by the legal requirements associated with these rights.

\section{Take down policy}

If you believe that this document breaches copyright please contact us (research.lib@cbs.dk) providing details, and we will remove access to the work immediately and investigate your claim. 
WP 2005-5

Term Structure Models with Parallel and

Proportional Shifts

by

Fredrik Armerin, Tomas Björk and Bjarne Astrup Jensen

INSTITUT FOR FINANSIERING, Handelshøjskolen i København

Solbjerg Plads 3, 2000 Frederiksberg C

tlf.: 38153615 fax: 38153600

DEPARTMENT OF FINANCE, Copenhagen Business School

Solbjerg Plads 3, DK - 2000 Frederiksberg C, Denmark

Phone (+45)38153615, Fax (+45)38153600

www.cbs.dk/departments/finance

ISBN 87-90705-96-3

ISSN 0903-0352 


\title{
Term Structure Models with Parallel and Proportional Shifts
}

\author{
Fredrik Armerin \\ Department of Mathematics \\ Royal Institute of Technology \\ SE-100 44 Stockholm, SWEDEN \\ armerin@math.kth.se
}

\author{
Bjarne Astrup Jensen, \\ Department of Finance
}

Copenhagen Business School

Solbjerg Plads 3

DK-2000 Frederiksberg, DENMARK

ba.fi@cbs.dk

\author{
Tomas Björk \\ Department of Finance, \\ Stockholm School of Economics, \\ Box 6501, \\ SE-113 83 Stockholm, SWEDEN \\ tomas.bjork@hhs.se
}

26th October 2005

\footnotetext{
${ }^{*}$ Financial support from the Danish Social Science Research Council is gratefully acknowledged.

${ }^{\dagger}$ The financial support of the Jan Wallander and Tom Hedelius Foundation is gratefully acknowledged.
} 


\begin{abstract}
We investigate the possibility of an arbitrage free model for the term structure of interest rates where the yield curve only changes through a parallel shift. We consider HJM type forward rate models driven by a multidimensional Wiener process as well as by a general marked point process. Within this general framework we show that there does indeed exist a large variety of nontrivial parallel shift term structure models, and we also describe these in detail. We also show that there exists no nontrivial flat term structure model. The same analysis is repeated for the similar case, where the yield curve only changes through proportional shifts.
\end{abstract}

Key words: bond market, term structure of interest rates, flat term structures. 


\section{Introduction}

The Macaulay or Redington duration measure for a fixed income portfolio, cf. the classical papers [18] and [21], uses the yield of the portfolio itself as the discount rate in calculating the weights. It is well known that this can only be a fully valid risk measure, comparable across different bonds and portfolios in a meaningful manner, if the term structure is flat and undergoes a parallel shift. Fisher and Weil showed in [13] that the assumption of a flat term structure could be discarded while maintaining the assumption of a parallel shift if one calculated the duration in accordance with weights derived from the actual term structure instead of an assumed flat term structure.

The issue of parallel shifts has been a recurrent one in the literature. It was noted by Ingersoll, Skelton and Weil in their seminal paper [16] from 1978 that a flat term structure and a noninfinitesimal parallel shift are incompatible with the "no arbitrage" requirement. This lack of consistency with the "no arbitrage" requirement has been noted by other authors in different model frameworks, see e.g. [8] and [19]. In [1], Armerin recently showed this in a rather general framework where the short term rate of interest was only assumed to be a continuous semimartingale.

Both before and after the publication of [16], numerous parameterized models for term structure shifts - often referred to as "shift functions" - have been introduced. " The historical background is well described by Ingersoll et. al. in [16]. Their analysis put some "order in chaos" by noting that for an already at that time voluminous literature on term structure changes little attention had been given to the question of compatibility with the "no arbitrage" requirement. They characterized the situation in the following way ([16], pp. 630-31):

"Largely missing, however, are any equilibrium considerations in modelling the stochastic process governing the yield structure. .... Fisher and Weil and other authors assumed additive shifts in the term structure that cannot occur in equilibrium in a competitive market. .... Many of those who assumed other types of changes in the yield curve also did so without considering whether such shifts can occur in equilibrium or do occur in fact."

Ingersoll et. al. also analyzed the issue of infinitesimal parallel shifts within the class of continuous time short rate models driven by a single Wiener process. They showed that for one particular choice of model, later known as the continuous time version of the Ho-Lee model, it is possible to have such infinitesimal parallel shifts for a term structure that is a second order polynomial with negative leading coefficient. Within this same class of models they also showed a particular arbitrage free model with Poisson input that allowed for parallel shifts through jumps. They referred to this possibility as "singular cases" ([16], p. 631 and p. 636, footnote 6):

\footnotetext{
"Except for the singular cases discussed in the Appendix, the occurence of non-infinitesimal, uniform changes in the yield curve implies arbitrage opportunities."

"As the example below indicates, changes in the yield curve can consist of constant noninfinitesimal shifts if they occur at any instant with vanishing small probability. However, in this case duration is not the proper risk measure. This issue is addressed in the Appendix."
}

A related issue concerns the classical textbook recipe for hedging an interest rate sensitive position: Use a hedge portfolio with (i) the same duration (ii) the same investment outlay and

\footnotetext{
${ }^{1}$ Representative examples of such models are found in, e.g., [15], [20] and [22].
} 
(iii) a higher level of convexity. This is an apparent arbitrage opportunity, and often portrayed as such, because no matter the direction of interest movements the hedge portfolio will always do better than the original portfolio - and the more realized interest rate volatility, the better. This puzzle has usually been explained away as an arbitrage opportunity by pointing to the parallel shift as an incorrect assumption. However, since parallel shifts are possible the resolution of the puzzle must be found elsewhere. In [10], Christensen and Sørensen used the parallel shift model from [16] and resolved the hedging puzzle by means of the partial differential equation (PDE) for short rate models. In option theoretic terms, the $\Delta$-value is analogous to the duration and the $\Gamma$-value is analogous to the convexity. Since the PDE ties the Greeks together, the higher level of convexity is paid for by a corresponding lower level of the $\theta$-value in portfolios with identical duration. Hence, the high convexity portfolio will loose (gain) value relative to the low convexity portfolio when interest rates show a low (high) level of realized volatility as time passes.

Our main object of study in this paper is an arbitrage free bond market in a very general continuous time setting, cf. [6]. We investigate if, when, and how it is possible to model an arbitrage free term structure of interest rates where the yield curve can only change by a parallel shift. We characterize the conditions under which such parallel shifts can occur using the approach of invariant manifolds and consistent forward curves developed in [5]. We show that the 2nd order polynomial example remains the only possibility in such a general environment, when the dynamics is driven by a multi-dimensional Wiener process. On the other hand; when jumps are allowed, infinitely many examples that allow for parallel shifts can be constructed, one of them being the Poisson-driven example from [16]. Finally, as an additional example we also characterize the possibilities under proportional shifts as originally introduced by Bierwag, Kaufman and Toevs in a multiple of publications, see e.g. [3] and [4].

The paper is organized as follows. In section 2 we introduce the notation used for the bond market. In section 3 we outline the general model for the dynamics of forward rates used in the paper. In section 4 the issue of parallel shifts and their compatibility with the general model used is analyzed. In section 5 we repeat the analysis for the case of proportional shifts. Finally, section 6 summarizes the conclusion of the paper.

\section{The Bond Market}

We denote the price at time $t$ of a pure discount bond maturing at time $t+x$ by $p(t, x)$. Note that we are using the Musiela parameterization, cf. [9], where $x$ denotes time to maturity, rather than the standard parametrization where $x$ typically denotes time of maturity. We define the various interest rates in the usual way.

\section{Definition 2.1}

- The yield $y_{t}(x)$ is defined by

$$
p(t, x)=e^{-y_{t}(x) x} \quad \Leftrightarrow \quad y_{t}(x)=-\frac{\ln p(t, x)}{x} .
$$

- The instantaneous forward rate at $t+x$, contracted at $t$, is defined by

$$
r_{t}(x)=-\frac{\partial \ln p(t, x)}{\partial x} .
$$


- The forward rate curve at time $t$, i.e. the mapping $x \longmapsto r_{t}(x)$, is denoted by $r_{t}$.

- The short rate $R_{t}$ is defined by

$$
R_{t}=r_{t}(0)
$$

- The money market account process is defined by

$$
B_{t}=e^{\int_{0}^{t} R_{s} d s} .
$$

We now go on to formally define the concept of a parallel shift term structure, as well as that of a flat term structure.

\section{Definition 2.2}

- A parallel shift term structure model is a term structure model where a.s.

$$
y_{t}(x)=\xi_{t}+H(x), \quad \forall(t, x) \in R_{+} \times R
$$

for some deterministic function $H: R_{+} \rightarrow R$ and some adapted process $\xi$.

- A flat term structure model is a term structure model where

$$
y_{t}(x)=\xi_{t}, \quad \forall(t, x) \in R_{+} \times R .
$$

In a parallel shift term structure model all yield curves are thus obtained by - possibly stochastic - parallel vertical shifts of the fixed yield curve $H$. In a flat yield curve model, all yield curves are flat, i.e. constant functions of the time to maturity $x$, and only change by parallel vertical shifts. Without loss of generality, we can normalize so that $H(0)=0$, and thus have the identification $\xi_{t}=R_{t}$.

Obviously, a deterministic term structure model, where the short rate $R_{t}$ is a deterministic constant, i.e. $y_{t}(x)=R_{t}$, is indeed a trivial case. Our goal is to investigate the characteristics of nontrivial parallel shift models. In order to carry out this investigation we need a formal framework to work in, and we now go on to present this.

\section{The Model}

We consider a financial market model living on a stochastic basis (filtered probability space) $(\Omega, \mathscr{F}, \mathbf{F}, Q)$ where $\mathbf{F}=\left\{\mathscr{F}_{t}\right\}_{t \geq 0}$. The basis is assumed to carry an $m$-dimensional Wiener process $W$ as well as a marked point process $\mu(d t, d z)$, on a measurable Lusin mark space $(Z, \mathscr{Z})$, with compensator $v(d t, d z)$. (See below for a more intuitive interpretation). We assume that $v([0, t] \times Z)<\infty P$-a.s. for all finite $t$, i.e. $\mu$ is a multivariate point process in the terminology of [17]. For simplicity we also assume that the compensator $v$ admits a predictable intensity measure, i.e. $v(d t, d z)=\lambda_{t}(d z) d t$. The bond market is assumed to be arbitrage free in the sense that the measure $Q$ is assumed to be an equivalent martingale measure. 
Remark 3.1 The intuitive interpretation of the point process $\mu$ is that we are modeling "events" which are occuring at discrete points in time, and a very concrete example could be the modeling of earth quakes or stock market crashes. As opposed to a more standard counting process setting, these discrete events are not all of the same type. Instead, every event is identified by its "mark" $z \in Z$, where the reader with very little loss of generality may think of $Z$ as $R^{n}$. In the earth quake example, a natural mark would be the strength of the earthquake on the Richter scale, and in this case the mark space $Z$ would be the positive real line $R_{+}$.

For term structure shifts, examples of the mark space could be the positive real line; or it could be interpreted as multiples of 5 basis points due to the central bank's discretionary changes of an "official short rate", $c f$. [2]. The informal interpretation of the point process $\mu$ is that $\mu$ is an integer valued (random) measure such that $\mu$ has a unit point mass at the point $(t, z) \in R_{+} \times Z$, if at time $t$ there is an event of the type $z$. The interpretation of the intensity measure $\lambda$ is - loosely speaking - that $\lambda_{t}(d z)$ is the expected number of events with marks in a "small set" $d z$, per unit time, conditional on the information in $\mathscr{F}_{t-}$. Thus the compensated point process $\tilde{\mu}(d t, d z)=\mu(d t, d z)-\lambda_{t}(d z) d t$ is "detrended" and possesses a natural martingale property. Note that the existence of an intensity rules out modeling discrete events occuring at deterministic points in time, i.e. events like "Alan Greenspan is making a speech November 23 at 12:15pm, at which point in time the term structure may change discretely". In more precise technical terms, an event cannot occur at an accessible stopping time.

We model the bond market by modeling the forward rate dynamics in the form of a HeathJarrow-Morton type model (see [14]) including jumps, as developed in [6]. To this end we need a proper space of forward rate curves to work in, and from [7] we borrow the following definition, which is needed for technical reasons, but which can be overlooked at a first reading. See also [12] for an extension of this functional analytical framework.

Definition 3.1 (The Space of Forward Rate Curves) Consider a fixed real number $\gamma>0$. The space $\mathscr{H}_{\gamma}$ is defined as the space of all infinitely differentiable functions

$$
r: R_{+} \rightarrow R
$$

satisfying the norm condition $\|r\|_{\gamma}<\infty$. Here the norm is defined as

$$
\|r\|_{\gamma}^{2}=\sum_{n=0}^{\infty} 2^{-n} \int_{0}^{\infty}\left(\frac{d^{n} r}{d x^{n}}(x)\right)^{2} e^{-\gamma x} d x .
$$

We now go on to define the forward rate dynamics, and to this end we have to specify the volatility structures for the Wiener as well as for the jump process. The particular value of $\gamma$ chosen in the definition of the space $\mathscr{H}_{\gamma}$ is irrelevant for the arguments to follow, so we simply drop this subscript from the notation.

- We consider a given Wiener volatility structure $\sigma$ of the form

$$
\sigma: \mathscr{H} \times R_{+} \rightarrow R^{m}
$$

In other words, each component $\sigma_{i}$ of the volatility vector $\sigma(r, x)=\left[\sigma_{1}(r, x), \ldots, \sigma_{m}(r, x)\right]$ is a real valued functional of the infinite dimensional $r$-variable, and a function of the real variable $x$. An alternative, and more profitable, way of viewing a component $\sigma_{i}$ is to see it 
as a mapping where a point $r \in \mathscr{H}$ (i.e. a forward rate curve) is mapped to the real valued function $\sigma_{i}(r, \cdot)$. We will in fact assume that this real valued function is a member of $\mathscr{H}$, which means that we can view each component $\sigma_{i}$ as a vector field $\sigma_{i}: \mathscr{H} \rightarrow \mathscr{H}$ on the space $\mathscr{H}$.

- We consider a given jump volatility structure $\delta$ of the form

$$
\delta: \mathscr{H} \times Z \times R_{+} \rightarrow R
$$

Thus $\delta(r, z, x)$ is a functional of the infinite dimensional $r$-variable, and a function of the $Z$-valued variable $z$ and of the real variable $x$. As for the Wiener volatility, we will instead consider $\delta$ from a more geometric point of view. Thus, for each $z \in Z$, we will view $\delta(\cdot, z, \cdot)$ as a mapping where the point $r \in \mathscr{H}$ is mapped to the real valued function $\delta(r, z, \cdot)$. We denote this mapping by $\delta_{z}$ and we assume that this real valued function is a member of $\mathscr{H}$, which means that for every fixed $z$ we can view $\delta_{z}$ as a vector field on $\mathscr{H}$, i.e. is a map $\delta_{z}: \mathscr{H} \rightarrow \mathscr{H}$.

We need some regularity assumptions, and the main ones are as follows. See [5] for technical details.

Assumption 3.1 We assume the following:

- For each $i=1, \ldots, m$ the volatility vector field $\sigma_{i}: \mathscr{H} \rightarrow \mathscr{H}$ is smooth.

- For each $z \in Z$ the vector field $\delta_{z}$ is smooth on $\mathscr{H}$.

- The intensity measure has the form $\lambda_{t}(d z)=\lambda\left(r_{t}, d z\right)$ where, for each fixed $r \in \mathscr{H}$, the intensity measure $\lambda(r, \cdot)$ is a deterministic nonnegative measure on $Z$.

The assumptions above will ensure that the forward rate process $r$ is an infinite dimensional Markov process, and we can now go on to specify the forward rate dynamics.

\section{Assumption 3.2 (Forward rate dynamics)}

We assume that the forward rates have dynamics of the following form.

$$
d r_{t}(x)=\alpha\left(r_{t}, x\right) d t+\sigma\left(r_{t}, x\right) d W_{t}+\int_{Z} \delta\left(r_{t}, z, x\right) \mu(d t, d z) .
$$

Remark 3.2 The interpretation of the point process integral above is the following: If at time $t$ we have the forward rate curve $r_{t}$, and there is an event of type $z$, then the forward rate with maturity $x$ will have a jump of size $\delta\left(r_{t}, z, x\right)$.

Since we are modeling directly under a martingale measure, we now need some sort of "drift condition" relating $\alpha$ to $\sigma$ and $\delta$. In [6] the standard HJM drift condition was extended to the case of a driving point process, and in the Musiela parameterization we easily (see [5]) obtain the following basic result. 
Proposition 3.1 (The Drift Condition) Under the assumption that the measure $Q$ is a martingale measure for the bond market, the following relation holds

$$
\alpha(r, x)=\frac{\partial}{\partial x} r(x)+\sigma(r, x) \int_{0}^{x} \sigma(r, s) d s-\int_{Z} \delta(r, z, x) e^{-D(r, z, x)} \lambda(r, d z),
$$

where

$$
D(r, z, x)=\int_{0}^{x} \delta(r, z, s) d s
$$

We thus have the following infinite dimensional SDE for the forward rates.

Proposition 3.2 (The Forward Rate Equation) Under the assumption that the measure $Q$ is a martingale measure for the bond market, the forward rate dynamics are given by the following equation

$$
\begin{aligned}
d r_{t}= & \left\{\mathbf{F} r_{t}+\mathbf{H} \sigma\left(r_{t}\right)-\int_{Z} \boldsymbol{\delta}\left(r_{t}, z\right) e^{-D\left(r_{t}, z\right)} \lambda\left(r_{t}, d z\right)\right\} d t \\
& +\sigma\left(r_{t}\right) d W_{t}+\int_{Z} \boldsymbol{\delta}\left(r_{t}, z\right) \mu(d t, d z) .
\end{aligned}
$$

Here $D$ is defined as above, the operator $\mathbf{F}$ is defined by

$$
\mathbf{F}=\frac{\partial}{\partial x}
$$

and the operator $\mathbf{H}$ is defined by

$$
\mathbf{H} \sigma(r, x)=\sigma(r, x) \int_{0}^{x} \sigma(r, s) d s .
$$

\section{Parallel Shifts}

We now go on to investigate the possibility of parallel shifts, and we start by noting that parallel shifts in the yield curve of the form

$$
y_{t}(x)=\xi_{t}+H(x),
$$

corresponds to parallel shifts of the forward rate curve of the form

$$
r_{t}(x)=\xi_{t}+h(x)
$$

where

$$
h(x)=\frac{d}{d x}\{H(x) \cdot x\}=H(x)+H^{\prime}(x) x .
$$

We note in passing that the earlier normalisation $H(0)=0$ implies $h(0)=0$. In vector space notation, this means that

$$
r_{t}=\xi_{t} \cdot e+h
$$

where the function $e \in \mathscr{H}$ is defined by

$$
e(x)=1, \quad \forall x \in R_{+} .
$$

We note that equation (12) says that the forward rate curve (viewed as a point in $\mathscr{H}$ ) always stays on the straight line in $\mathscr{H}$, which passes through the point $h$ and has direction $e$. We thus have the following result. 
Proposition 4.1 The model admits parallel shifts if and only if the the linear manifold $\mathscr{M}$ defined by

$$
\mathscr{M}=h+R \cdot e,
$$

is invariant under the action of the forward rate equation.

In the language of [5] this means that the model admits parallel shifts if and only if the forward rate equation is consistent with the manifold $\mathscr{M}$. We can thus use the results from [5] concerning consistency, but this requires us to write the forward rate equation on Stratonovich form. This is easily done, and we recall the following result from [5].

\section{Proposition 4.2 (The Forward Rate Equation: Stratonovich Form)}

The forward rate dynamics of (3.2) can equivalently be written on Stratonovich form as are given by the following equation

$$
\begin{aligned}
d r_{t}= & \left\{\mathbf{F} r_{t}+\mathbf{H} \boldsymbol{\sigma}\left(r_{t}\right)-\frac{1}{2} \sigma^{\prime}\left(r_{t}\right) \boldsymbol{\sigma}\left(r_{t}\right)-\int_{Z} \delta\left(r_{t}, z\right) e^{-D\left(r_{t}, z\right)} \lambda\left(r_{t}, d z\right)\right\} d t \\
& +\sigma\left(r_{t}\right) \circ d W_{t}+\int_{Z} \delta\left(r_{t}, z\right) \mu(d t, d z),
\end{aligned}
$$

where o denotes the Stratonovich integral and $\sigma^{\prime}(r)$ denotes the Fréchet derivative of $\sigma$ w.r.t. the variable $r$.

We can now cite Proposition 6.2 from [5], which is the main result we will use concerning consistency.

Proposition 4.3 The manifold $\mathscr{M}$ is invariant under the forward rate equation if and only if, at very point of $\mathscr{M}$, the drift and volatility vector fields of the Stratonovich forward rate equation all belong to the tangent space of $\mathscr{M}$. In other words; we have invariance if and only if the following collection of vector fields all belong to the tangent space of $\mathscr{M}$.

$$
\begin{aligned}
& \mathbf{F} r+\mathbf{H} \boldsymbol{\sigma}(r)-\frac{1}{2} \sigma^{\prime}(r) \sigma(r)-\int_{Z} \delta(r, z) e^{-D(r, z)} \lambda(r, d z), \\
& \sigma_{i}(r), \quad i=1, \ldots m, \\
& \delta_{z}(r), \quad \forall z \in Z .
\end{aligned}
$$

We will now apply this result to our particular case, where $\mathscr{M}$ is given by (13) above, but in order to proceed we have to identify the tangent space $T_{\mathscr{M}}(r)$ of $\mathscr{M}$ at each point $r \in \mathscr{M}$. This turns out to be very easy: since $\mathscr{M}$ is a linear manifold, the tangent space is spanned by the vector $e$, so we have

$$
T_{\mathscr{M}}(r)=R \cdot e, \quad \forall r \in \mathscr{M} .
$$

Thus; the invariance conditions from Proposition 4.3, applied to our case, read as follows.

$$
\begin{aligned}
& \mathbf{F} r+\mathbf{H} \sigma(r)-\frac{1}{2} \sigma^{\prime}(r) \sigma(r)-\int_{Z} \delta(r, z) e^{-D(r, z)} \lambda(r, d z)=\psi(r) \cdot e, \\
& \sigma_{i}(r)=\varphi_{i}(r) \cdot e, \quad i=1, \ldots m \\
& \delta_{z}(r)=\gamma_{z}(r) \cdot e, \quad \forall z \in Z
\end{aligned}
$$


for some scalar fields $\psi, \varphi_{i}$, and $\gamma_{z}$, where we will use either the notation $\gamma_{z}(r)$ or the notation $\gamma(r, z)$, depending on the context. We note that since $e$ is constant as a function of $x$, this means, in more pedestrian terms, that the objects on the left hand sides of the equations above do not depend upon $x$. Furthermore, since each $\sigma_{i}$ maps $\mathscr{H}$ into the subspace $T_{\mathscr{M}}$, this also holds for the Fréchet derivative $\sigma^{\prime}(r)$. Thus (18) can be replaced by the condition

$$
h^{\prime}+\mathbf{H} \sigma(r)-\int_{Z} \delta(r, z) e^{-D(r, z)} \lambda(r, d z)=\psi(r) \cdot e,
$$

where we have used the fact that, for an $r \in \mathscr{M}$ we have $r=h+\xi e$ for some scalar $\xi$, so we have

$$
\mathbf{F} r=\frac{\partial}{\partial x}(h+\xi e)=h^{\prime}
$$

Written out in detail, (19)-(20) simply says that

$$
\begin{aligned}
\sigma_{i}(r, x) & =\varphi_{i}(r), \quad i=1, \ldots m, \\
\delta(r, z, x) & =\gamma(r, z), \quad \forall z \in Z,
\end{aligned}
$$

After substituting this into (21) we obtain, after trivial calculations

$$
h^{\prime}(x)+\left(\sum_{i=1}^{m} \varphi_{i}^{2}(r)\right) x-\int_{Z} \gamma(r, z) e^{-\gamma(r, z) x} \lambda(r, d z)=\psi(r) .
$$

Integrating this in $x$ over the interval $[0, x]$, assuming enough regularity to allow an application of the Fubini Theorem, and recalling that $h(0)=0$, we obtain

$$
h(x)=-\frac{1}{2}\left(\sum_{i=1}^{m} \varphi_{i}^{2}(r)\right) x^{2}+\psi(r) x+\int_{Z}\left\{1-e^{-\gamma(r, z) x}\right\} \lambda(r, d z) .
$$

We can now finally formulate and prove our main result.

Theorem 4.1 For the general model framework defined in section 3 the following is true:

1. There does indeed exist nontrivial arbitrage free parallel shift term structure models.

2. The following conditions are necessary for the existence of a parallel shift term structure model

- The Wiener forward rate volatilities must be independent of the maturity variable $x$, i.e. they must have the form

$$
\sigma_{i}(r, x)=\varphi_{i}(r), \quad i=1, \ldots m,
$$

and furthermore, the squared sum

$$
B^{2}=\sum_{i=1}^{m} \varphi_{i}^{2}(r)
$$

must be constant as a function of $r$. 
- The jump volatility $\delta$ must be independent of the maturity variable $x$, i.e. it must be of the form

$$
\delta(r, z, x)=\gamma(r, z)
$$

- The integral

$$
\int_{Z}\left\{1-e^{-\gamma(r, z) x}\right\} \lambda(r, d z)
$$

must be constant as a function of $r$.

3. If a forward rate model admits a parallel shift term structure of the form

$$
r_{t}(x)=R_{t}+h(x)
$$

then $h$ must have the form

$$
h(x)=-\frac{1}{2} B^{2} x^{2}+C x+\int_{Z}\left\{1-e^{-\gamma(z) x}\right\} \lambda(d z),
$$

where $B, C$ are constants, $\gamma(z)$ is a real valued function on $Z$, and $\lambda(d z)$ is a positive finite measure on $Z$.

4. The conditions above are also sufficient. More precisely: for every hiven by (31) above, there exists a corresponding parallel shift forward rate model, admitting the particular $h$ as the shift function. The forward rate model is obtained by choosing constants $\varphi_{1}, \ldots, \varphi_{m}$, such that

$$
B^{2}=\sum_{i=1}^{m} \varphi_{i}^{2},
$$

and then defining the jump intensity and the forward rate volatilities by the prescription

$$
\begin{aligned}
\sigma_{i}(r, x) & =\varphi_{i}, \quad i=1, \ldots, m, \\
\delta(r, z, x) & =\gamma(z), \\
\lambda(r, d z) & =\lambda(d z) .
\end{aligned}
$$

Proof. The items 1, 3 and 4 in the statement of the Theorem above are clear from the earlier arguments. It remains only to prove the necessary conditions. These however, follows immediately from equation (25), plus the observation that since the right hand side does not depend upon $r$, and since the functions $x, x^{2}$, and $\left\{e^{-c x} ; c \in R\right\}$ are linearly independent, the coefficients in front of $x$ and $x^{2}$ must be independent of $r$. Thus the integral term in (29) must also be independent of $r$.

For the purely Wiener driven case we have the following corollary, which is an extension of the corresponding result in [16] and [10], where the study a priori was restricted to short rate models with constant coefficients and a single driving Wiener process.

Corollary 4.1 The only way to obtain a parallel shift term structure model for a purely Wiener driven model is when the shift function is a quadratic function with negative leading term, and the forward rate model is the Ho-Lee model. 
Proof. For a purely Wiener driven model we have, from (31),

$$
h(x)=-\frac{1}{2} B^{2} x^{2}+C x
$$

with

$$
B^{2}=\sum_{i=1}^{m} \varphi_{i}^{2}(r) .
$$

This proves that the only possible shape of the shift function is quadratic. Furthermore, we can define a scalar Wiener process $\hat{W}$ by

$$
d \hat{W}_{t}=\frac{1}{B} \sum_{i=1}^{m} \varphi_{i}(r) d W_{t}^{i},
$$

and write the forward rate dynamics as

$$
d r_{t}(x)=\left\{\mathbf{F} r_{t}(x)+B^{2} x\right\} d t+B d \hat{W}_{t}=C d t+B d \hat{W}_{t}
$$

Thus we have a forward rate model with constant volatility, which we recognize as the Ho-Lee short rate model, written in forward rate form.

In addition to the result above we also have an easy corollary concerning the non-existence of flat term structure models.

Corollary 4.2 The only way to obtain a flat term structure model is by having a deterministic constant short rate.

Proof. A flat term structure implies that the function $h(x)=H(x)+H^{\prime}(x) x$ is a constant. From (25) it then follows that $\varphi_{1}, \ldots, \varphi_{m}$ and $\gamma(z)$ all have to be zero, i.e. we have a zero volatility model.

Parallel shift models are thus very restricted indeed when jumps are excluded. On the other hand, parallel shift models with jumps are in no way "singular cases". An abundance of models can be constructed in our general framework using compound Poisson processes, Cox processes and other jump processes now commonly applied in finance. A particular simple example is the model presented in [16] with only one driving Poisson process with constant intensity $\lambda$ and a constant $\gamma$-function. In this case, we have from (31) (with $B=C=0$ ):

$$
h(x)=\lambda\left(1-e^{-\gamma x}\right),
$$

from the relation $h(x)=H(x)+H^{\prime}(x) x$ we may integrate, and use the normalisation $H(0)=0$ to obtain

$$
y_{t}(x)=R_{t}+\lambda\left(1-\frac{1-e^{-\gamma x}}{\gamma x}\right) .
$$

Note that $h$ above is not the only possible shift function for this model; all shift functions of the form

$$
h(x)=C x+\lambda\left(1-e^{-\gamma x}\right)
$$

for an arbitrary choice of $C$ are in fact supported by the model. 


\section{Proportional shifts}

The proportional shift model has been another model frequently referred to in the duration literature (see [3] and [4]). A proportional shift term structure model is a term structure model where a.s.

$$
y_{t}(x)=\xi_{t} H(x), \quad \forall(t, x) \in R_{+} \times R
$$

for some deterministic function $H: R_{+} \rightarrow R$ and some adapted process $\xi$.

In a proportional shift model all yield curves are thus obtained by - possibly stochastic proportional vertical shifts of the fixed yield curve $H$. Without loss of generality one can choose $H(0)=1$ and $\xi_{t}=R_{t}$.

It is straightforward to see that for a proportional shift model, the forward rates are given by

$$
r_{t}(x)=\xi_{t} h(x)
$$

where, as before, $h(x)=\frac{d}{d x}\{H(x) x\}$, and we have the normalization $\xi_{t}=R_{t}$ and $h(0)=1$. The invariant manifold for the forward rate curve is thus given by the subspace

$$
\mathscr{M}=R \cdot h,
$$

so the tangent space is spanned by the vector $h$, and we have

$$
T_{\mathscr{M}}(r)=R \cdot h, \quad \forall r \in \mathscr{M} .
$$

Thus the invariance conditions from Proposition 4.3 read as follows:

$$
\begin{aligned}
& \mathbf{F} r+\mathbf{H} \sigma(r)-\frac{1}{2} \sigma^{\prime}(r) \sigma(r)-\int_{Z} \delta(r, z) e^{-D(r, z)} \lambda(r, d z)=\psi(r) \cdot h, \\
& \sigma_{i}(r)=\varphi_{i}(r) \cdot h, \quad i=1, \ldots m, \\
& \delta(r, z)=\gamma_{z}(r) \cdot h, \quad \forall z \in Z,
\end{aligned}
$$

for some scalar fields $\psi, \varphi_{i}$, and $\gamma_{z}$.

Following the analysis in section 4 the function $h(x)$ must satisfy the condition

$$
\xi h^{\prime}+\mathbf{H} \boldsymbol{\sigma}(r)-\int_{Z} \delta(r, z) e^{-D(r, z)} \lambda(r, d z)=\psi(r) \cdot h .
$$

where we have used the fact that, for an $r \in \mathscr{M}$ we have $r=\xi h$ for some scalar $\xi$, so we have

$$
\mathbf{F} r=\frac{\partial}{\partial x}(\xi h)=\xi h^{\prime}
$$

Written out in detail, (47)-(48) simply says that

$$
\begin{aligned}
\sigma_{i}(r, x) & =\varphi_{i}(r) h(x), \quad i=1, \ldots m, \\
\delta(r, z, x) & =\gamma_{z}(r) h(x), \quad \forall z \in Z .
\end{aligned}
$$

After substituting this into (49) we obtain, after trivial calculations

$$
\xi h^{\prime}(x)+\left(\sum_{1}^{m} \varphi_{i}^{2}(r)\right) h(x) V(x)-\int_{Z} \gamma(z, r) h(x) e^{-\gamma(r, z) V(x)} \lambda(r, d z)=\psi(r) h(x),
$$


where

$$
V(x)=\int_{0}^{x} h(s) d s .
$$

Integrating this in $x$ over the interval $[0, x]$, using the normalizations $h(0)=1$ and $\xi=R=r(0)$, and again assuming enough regularity to allow an application of the Fubini Theorem, gives us

$$
\begin{aligned}
R\left(V^{\prime}(x)-1\right)= & -\frac{1}{2}\left(\sum_{i=1}^{m} \varphi_{i}^{2}(r)\right) V^{2}(x)+\psi(r) V(x) \\
& -\int_{Z}\left\{1-e^{-\gamma(r, z) V(x)}\right\} \lambda(r, d z) .
\end{aligned}
$$

As long as $R>0$ we can divide through and get

$$
\begin{aligned}
V^{\prime}(x)-1= & -\frac{1}{2}\left(\frac{\sum_{1}^{m} \varphi_{i}^{2}(r)}{R}\right) V^{2}(x)+\frac{\psi(r)}{R} V(x) \\
& -\int_{Z}\left\{1-e^{-\gamma(r, z) V(x)}\right\} \frac{1}{R} \lambda(r, d z) .
\end{aligned}
$$

We can now formulate the result for this section.

Theorem 5.1 For the model framework defined in section 5 the following is true:

1. There does indeed exist nontrivial arbitrage free proportional shift term structure models.

2. The following conditions are necessary for the existence of a proportional shift structure.

- The forward rate volatilities must be proportional to the shift function h, i.e. they must have the form

$$
\begin{aligned}
\sigma_{i}(r, x) & =\varphi_{i}(r) h(x), \quad i=1, \ldots m, \\
\delta(r, z, x) & =\gamma(r, z) h(x), \quad \forall z \in Z .
\end{aligned}
$$

- Furthermore, there must exist constants a and $\sigma$ such that for all $r$

$$
\begin{aligned}
\sum_{i=1}^{m} \varphi_{i}^{2}(r) & =\sigma^{2} r(0), \\
\psi(r) & =\operatorname{ar}(0) .
\end{aligned}
$$

- The integral

$$
\int_{Z}\left\{1-e^{-\gamma(r, z) V(x)}\right\} \frac{1}{r(0)} \lambda(r, d z)
$$

must be independent of $r$.

3. If the model admits a proportional shift term structure of the form

$$
r_{t}(x)=R_{t} h(x)
$$

then $V(x)=\int_{0}^{x} h(s) d s$ must satisfy an integro differential equation of the form

$$
V^{\prime}(x)+\frac{1}{2} \sigma^{2} V(x)^{2}-a V(x)+\int_{Z}\left\{1-e^{-\gamma(z) V(x)}\right\} \lambda(d z)=1,
$$

where $\sigma$ and a are arbitrary constants, $\gamma(z)$ is a real valued function on $Z$, and $\lambda(d z)$ is a positive finite measure on $Z$. 
4. For every $h \geq 0$ given by (60) above and every non-negative value of the short rate $r(0)$, there exists a corresponding proportional shift forward rate model, admitting the particular $h$ as the shift function. The forward rate model is obtained by choosing functions $\varphi_{1}(r), \ldots, \varphi_{m}(r)$, such that

$$
\sum_{i=1}^{m} \varphi_{i}^{2}(r)=\sigma^{2} r(0)
$$

and the defining the jump intensity and the forward rate volatilities by the prescription

$$
\begin{aligned}
\sigma_{i}(r, x) & =\varphi_{i} \sqrt{r(0)} h(x), \quad i=1, \ldots, m, \\
\delta(r, z, x) & =\gamma(z) h(x), \\
\lambda(r, d z) & =\lambda(d z) .
\end{aligned}
$$

Proof. The proof follows almost exactly the proof of Theorem 4.1.

We note that the differential equation (60) for $V$ above has the form of a standard Riccati equation, apart from the integral term connected to the jump part of the forward rate process. In general it is of course not possible to obtain an analytical solution of equation (60), but there are some simple special cases which we now go on to discuss.

Firstly; for the purely Wiener driven case we have the following simple result:

Corollary 5.1 The only way to obtain a proportional shift term structure model for a purely Wiener driven model is when the shift function is a solution to a Riccati equation. This forward rate model is the CIR-model with mean reversion level $0 ;$ hence, it will eventually be absorbed at 0 a.s.

Proof. If the jump term is not present, then equation (60) is a standard Riccati equation whose solution is well known:

$$
\begin{aligned}
V(x) & =\frac{2\left(e^{\mu x}-1\right)}{(\mu-a)\left(e^{\mu x}-1\right)+2 \mu} \\
\mu & =\sqrt{a^{2}+2 \sigma^{2}}
\end{aligned}
$$

This is a special case of the CIR model with mean reversion level 0:

$$
d R_{t}=a R_{t} d t+\sigma \sqrt{R_{t}} d W_{t}
$$

for which $R_{t}$ - and, hence, the entire term structure - will be absorbed at zero at some point in time a.s.

Secondly; let us consider the pure Poisson case with intensity $\lambda$. Then, setting $a=\sigma=0$, equation (60) takes the form

$$
V^{\prime}(x)+\left(1-e^{-\gamma V(x)}\right) \lambda=1
$$


Substituting $g(x) \equiv e^{-\gamma V(x)}$ we get, after trivial calculations, the following differential equation for $g(x)$ :

$$
g^{\prime}(x)=-\lambda \gamma g(x)^{2}+(\lambda-1) \gamma g(x), g(0)=1 .
$$

This is easily solved by separation of variables:

$$
g(x)= \begin{cases}\frac{(1-\lambda) e^{\gamma(1-\lambda) x}}{1-\lambda e^{\gamma(1-\lambda) x},} & \lambda \neq 1 \\ \frac{1}{1+\gamma x}, & \lambda=1 .\end{cases}
$$

From (70), $h(x)$ is obtained by substitution:

$$
h(x)=V^{\prime}(x)=(1-\lambda)+\lambda g(x)= \begin{cases}\frac{(1-\lambda)}{1-\lambda e^{\gamma(1-\lambda) x},} & \lambda \neq 1, \\ \frac{1}{1+\gamma x}, & \lambda=1 .\end{cases}
$$

\section{Conclusions}

Term structure models with certain stylized types of shifts have attracted much attention in the literature on duration and risk measurement for bond portfolios. The issue of parallel shifts has been a recurrent one and underlies such risk measures as the Macaulay duration and the FisherWeil duration in widespread practical use. Similarly, proportional shifts have been introduced in a number of earlier papers on duration.

As pointed out already by Ingersoll, Skelton and Weil in their seminal paper [16] from 1978, little attention is paid in this literature to equilibrium considerations in the modelling of term structure movements. A modelling practice, where suitable stochastic processes and "shift functions" are chosen on an ad hoc basis to produce seemingly reliable risk measures, is still commonly used.

This paper re-examines the parallel and proportional types of shifts in a fairly general continuous time setup by applying the concepts of consistent forward curves and invariant manifolds developed in [5]. We demonstrate that models with parallel and proportional shifts are indeed very restricted when the dynamics is driven by a multi-dimensional Wiener process. For the parallel case, only a specific version of the Ho-Lee model, where the term structure is a secondorder polynomial with negative leading coefficient, is possible. For the proportional case, only a specific version of the CIR model is possible. This version is degenerate in the sense that zero is an absorbing boundary for the entire term structure, which is reached a.s. at some point in time.

However, when the modelling framework is extended to include a marked point process a large variety of possible term structure models that allows for these two types of stylized shifts arises. This variety of possible term structure curves does not constitute "singular cases", cf. the quotation from [16] mentioned above, but analytical examples of such term structure models are limited by the ability to calculate the integral term related to the marked point process. 


\section{References}

[1] Armerin, F. Aspects of cash flow valuation. PhD thesis, Department of Mathematics, The Royal Institute of Technology, Stockholm, Sweden, Dec. 2004.

[2] BabBs, S., AND WeBbER, N. Term structure modeling under alternative official regimes. 1997 , pp. 394-422 in [11].

[3] Bierwag, G. O., And Kaufman, G. G. Immunization, duration and the term structure of interest rates. Journal of Financial and Quantitative Analysis (1977).

[4] Bierwag, G. O., Kaufman, G. G., And Toevs, A. Duration: Its development and use in bond portfolio management. Financial Analysts Journal, July-August (1983), 15-35.

[5] BJÖRK, T., AND CRISTENSEN, B. Interest rate dynamics and consistent forward rate curves. Mathematical Finance 9, 4 (1999), 323-348.

[6] Björk, T., KabAnov, Y., And Runggaldier, W. Bond market structure in the presence of a marked point process. Mathematical Finance 7, 2 (1997), 211-239.

[7] BJörk, T., AND SVEnsson, L. On the existence of finite dimensional realizations for nonlinear forward rate models. Mathematical Finance 11, 2 (2001), 205-243.

[8] Boyle, P. P. Immunization under stochastic models of the term structure. Journal of the Institute of Actuaries 105 (1978), 177-187.

[9] Brace, A., Gatarek, D., And Musiela, M. The market model of interest rate dynamics. Mathematical Finance 7, 2 (1997), 127-154.

[10] Christensen, P. O., And Sørensen, B. G. Duration, convexity and time value. Journal of Portfolio Mangement (1994), 51-60.

[11] Dempster, M., And Pliska, S. R., Eds. Mathematics of Derivative Securities. Publications of the Newton Institute. Cambridge University Press, Cambridge, U.K., 1997.

[12] Filipović, D., And Teichmann, J. Existence of finite dimensional realizations for stochastic equations, 2001. Forthcoming in Journal of Functional Analysis

[13] FISHER, L., AND WEIL, R. L. Coping with the risk of interest rate fluctuations: Return to bondholders from naive and optimal strategies. Journal of Business 44 (1971), 408-431.

[14] Heath, D., Jarrow, R., And Morton, A. Bond pricing and the term structure of interest rates: a new methodology for contingent claims valuation. Econometrica 60 (1992), 77-105.

[15] HÜrlimann, W. On immunization, stop-loss order and the maximum Shiu measure. Insurance: Mathematics and Economics 31 (2002), 315-325.

[16] Ingersoll, JR., J. E., Skelton, J., And Weil, R. L. Duration forty years later. Journal of Financial and Quantitative Analysis 13, 4 (1978), 627-650.

[17] JACOD, J., AND ShIRYAEV, A. Limit Theorems for Stochastic Processes. Springer Verlag, 1987. 
[18] MaCAulay, F. Some theoretical problems suggestes by the movements of interest rates, bond yields, and stock prices in the U.S. since 1856. NBER, New York, USA, 1938.

[19] Milgrom, P. R. Measuring the interest rate risk. Transactions of the Society of Actuaries 37 (1985), 241-257.

[20] Montrucchio, L., And L., P. A note on Shiu-Fisher-Weil immunization theorem. Insurance: Mathematics and Economics 10 (1991), 125-131.

[21] Redington, F. Review of the principles of life-office valuations. Journal of the Institute of Actuaries (1952), 286-340.

[22] ShIU, E. S. W. On Redington's theory of immunization. Insurance: Mathematics and Economics 9, 4 (1990), 171-175. 
INSTITUT FOR FINANSIERINGs working papers siden 2000:

WP 2000-1 Bjarne Astrup Jensen and Carsten Sørensen: Paying for minimum interest rate guarantees: Who should compensate who?

WP 2000-2 Jan Jakobsen and Ole Sørensen: Decomposing and testing Long-run Returns with an application to initial public offerings in Denmark.

WP 2000-3 Jan Jakobsen and Torben Voetmann: Volatility-Adjusted Performance An Alternative Approach to Interpret Long-Run Returns.

WP 2000-4 Jan Jakobsen and Torben Voetmann: Post-Acquisition Performance in the Short and Long-Run Evidence from the Copenhagen Stock Exchange 1993-1997.

WP 2000-5 Ken L. Bechmann and Johannes Raaballe: A Regulation of Bids for Dual Class Shares. Implication: Two Shares - One Price.

WP 2000-6 Torben Voetmann: Changes in the Bid-Ask Componets Around Earnings Announcemtents: Evidence from the Copenhagen Stock Exchange.

WP 2000-7 Henrik Lando: The Optimal Standard of Proof in Criminal Law When Both Fairness and Deterrence Are Social Aims.

WP 2000-8 Jesper Rangvid and Carsten Sørensen: Convergence in the ERM and Declining Numbers of Common Stochastic Trends.

WP 2000-9 Claus Munk and Carsten Sørensen: Optimal Consumption and Investment Strategies with Stochastic Interest Rates.

WP 2000-10 Henrik Lando and Caspar Rose: On Specific Performance in Civil Law and Enforcement Costs.

WP 2000-11 Henrik Lando: Ny lov om jordforurening i økonomisk belysning.

WP 2001-1 Michael Møller, Claus Parum og Thomas Sørensen: Den ny pensionsafkastbeskatningslov.

WP 2001-2 Bjarne Astrup Jensen: Mean variance efficient portfolios by linear programming: A review of some portfolio selection criteria of Elton, Gruber and Padberg. 
WP 2001-3 Caspar Rose: Impact of Investor Meetings/Presentations on Share Prices, Insider Trading and Securities Regulation.

WP 2001-4 Caspar Rose: Corporate Financial Performance and the Use of Takeover Defenses.

WP 2001-5 Shubhashis Gangopadhyay and Clas Wihlborg: The Impact of Bankruptcy Rules on Risky Project Choice and Skill Formation under Credit Rationing.

WP 2001-6 Claus Munk, Carsten Sørensen \& Tina Nygaard Vinther: Portfolio Choice under Inflation: Are Popular Recommendations Consistent with Rational Behaviour?

WP 2001-7 Ken L. Bechmann: Evidence on the Limits of Arbitrage: Short Sales, Price Pressure, and the Stock Price Response to Convertible Bond Calls.

WP 2001-8 Michael Møller \& Caspar Rose: Legal pre-emption rights as calloptions, redistribution and efficiency loss.

WP 2001-9 Peter Raahauge: Empirical Rationality in the Stock Market.

WP 2002-1 Bjarne Astrup Jensen: On valuation before and after tax in no arbitrage models: Tax neutrality in the discrete time model.

WP 2002-2 Ken L. Bechmann: Price and Volume Effects Associated with Changes in the Danish Blue-Chip Index - The KFX Index.

WP 2002-3 Steen Thomsen and Caspar Rose: Foundation ownership and financial performance. Do companies need owners?

WP 2002-4 Martin Richter and Carsten Sørensen: Stochastic Volatility and Seasonality in Commodity Futures and Options: The Case of Soybeans.

WP 2002-5 Caspar Rose: Aktiemarkedets reaktion på indførelsen af incitamentsprogrammer.

WP 2002-6 Caspar Rose: Impact of Takeover Defenses on Managerial Incentives.

WP 2002-7 Ken L. Bechmann og Peter Løchte Jørgensen: Optionsaflønning i danske børsnoterede selskaber.

WP 2002-8 Jesper Rangvid: Output and Expected Returns - a multicountry study. 
WP 2002-9 Jonas Aziz Bhatti og Michael Møller: Pensionsafkastbeskatning og optimal porteføljesammensætning.

WP 2003-1 Bjarne Florentsen, Michael Møller and Niels Chr. Nielsen: Reimbursement of VAT on written-off Receivables.

WP 2003-2: Ken L. Bechmann and Peter Løchte Jørgensen: The Value and Incentives of Option-based Compensation in Danish Listed Companies.

WP 2004-1 Ken L. Bechmann and Johannes Raaballe: The Difference Between Stock Splits and Stock Dividends - Evidence from Denmark.

WP 2004-2 Caspar Rose: Bestyrelsessammensætning og finansiel performance i danske børsnoterede virksomheder - Er Nørbyrapportens anbefalinger til gavn for aktionærerne?

WP 2004-3 Jens Lunde: Lack of balance in after-tax returns - lack of tenure neutrality. The Danish case.

WP 2004-4 Peter Raahauge: Upper Bounds on Numerical Approximation Errors.

WP 2004-5 Peter Raahauge: Higher-Order Finite Element Solutions of Option Prices.

WP 2004-6 Anders Holst \& Morten Nalholm: Modelling Callable Annuity Bonds with Interest-Only Optionality.

WP 2005-1 Finn Østrup: Fiscal Policy and Welfare under Different Exchange Rate Regimes.

WP 2005-2 Finn Østrup: The Choice of Monetary Regime: Fiscal Implications.

WP 2005-3 Lunde, Jens: The owner-occupiers’ structure during a house price boom - Does negative equity exist as a permanent feature in the Danish housing market?

WP 2005-4 Ken L. Bechmann and Johannes Raaballe: Taxable Cash Dividends - A Useful Way of Burning Money. 
WP 2005-5 Bjarne Astrup Jensen, Fredrik Armerin and Tomas Björk: Term Structure Models with Parallel and Proportional Shifts. 